\title{
Racial/ethnic and immigrant differences in early childhood diet quality
}

\author{
Marieke LA de Hoog 1,2,*, Ken P Kleinman ${ }^{3}$, Matthew W Gillman ${ }^{3,4}$, \\ Tanja GM Vrijkotte ${ }^{1}$, Manon van Eijsden ${ }^{2,5}$ and Elsie M Taveras ${ }^{3,6}$ \\ 'Department of Public Health, Academic Medical Centre, University of Amsterdam, PO Box 22660, 1100 DD \\ Amsterdam, The Netherlands: ${ }^{2}$ Department of Epidemiology, Documentation and Health Promotion, Public \\ Health Service, Amsterdam, The Netherlands: ${ }^{3}$ Obesity Prevention Program, Department of Population Medicine, \\ Harvard Medical School and Harvard Pilgrim Health Care Institute, Boston, MA, USA: ${ }^{4}$ Department of Nutrition, \\ Harvard School of Public Health, Boston, MA, USA: ${ }^{5}$ Institute of Health Sciences, VU University, Amsterdam, \\ The Netherlands: 'Division of General Pediatrics, Children's Hospital Boston, Boston, MA, USA
}

Submitted 30 July 2012: Final revision received 10 March 2013: Accepted 17 March 2013: First published online 7 May 2013

\begin{abstract}
Objective: To assess racial/ethnic differences in the diet in young children and the explanatory role of maternal BMI, immigrant status and perception of child's weight. Design: Among white, black and Hispanic 3-year-olds, we used negative binomial and linear regression to examine associations of race/ethnicity with foods and nutrients assessed by a validated FFQ.

Setting: Project Viva, Boston (MA), USA.

Subjects: Children aged 3 years ( $n$ 898).

Results: Mean age was 38.3 (sD 2.8) months; 464 (52\%) were boys and 127 mothers (14\%) were immigrants. After adjustment for sociodemographic factors, black and Hispanic children ( $v$. white) had a higher intake of sugar-sweetened beverages $($ rate ratio $(\mathrm{RR})=2.59(95 \% \mathrm{CI} 1.95,3.48)$ and $\mathrm{RR}=1.59(95 \% \mathrm{CI} 1.07,2.47)$, respectively) and lower intakes of skimmed $/ 1 \%$ milk $(\mathrm{RR}=0.42(95 \% \mathrm{CI} 0.33,0.53)$ and $\mathrm{RR}=0.43(95 \% \mathrm{CI} 0.31,0.61)$, respectively) and trans-fat $(-0.10(95 \% \mathrm{CI}$ $-0 \cdot 18,-0 \cdot 03) \%$ of energy and $-0 \cdot 15(95 \%$ CI $-0 \cdot 26,-0 \cdot 04) \%$ of energy, respectively). Among Hispanics only, a lower intake of snack food ( $R R=0.83(95 \%$ CI $0.72,0.98)$ ) was found and among blacks only, a higher intake of fast food $(\mathrm{RR}=1 \cdot 28(95 \% \mathrm{CI} 1 \cdot 05,1 \cdot 55))$ and lower intakes of saturated fat $(-0 \cdot 86(95 \% \mathrm{CI}$ $-1.48,-0.23) \%$ of energy), dietary fibre $(0.85(95 \%$ CI $0.08,1.62) \mathrm{g} / \mathrm{d})$ and Ca $(-120(95 \% \mathrm{CI}-175,-65) \mathrm{mg} / \mathrm{d})$ were found. Being born outside the USA was associated with more healthful nutrient intakes and less fast food.

Conclusions: Three-year-old black and Hispanic ( $v$. white) children ate more sugarsweetened beverages and less low-fat dairy. Total energy intake was substantially higher in Hispanic children. Snack food (Hispanic children) and fat intakes (black children) tended to be lower. Children of immigrants ate less fast food and bad fats and more fibre.
\end{abstract}

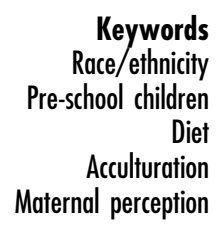

Many racial/ethnic minority groups living in Western societies have higher rates of obesity compared with whites, even among children ${ }^{(1-5)}$. Results from the National Health and Nutrition Examination Survey (NHANES) showed that the prevalence of obesity (BMI $\geq 95$ th percentile) in 2-5-year-old children was highest among blacks $(17 \cdot 0 \%)$ and Hispanics (14.6\%) compared with whites $(6 \cdot 0 \%)^{(2)}$. Given the strong tracking of childhood BMI levels into adulthood ${ }^{(6)}$, it is plausible that these racial/ ethnic differences in childhood overweight increase the risk for developing several chronic diseases in adulthood ${ }^{(7)}$.

The aetiology of obesity is complex, but is ultimately determined by the long-term balance between energy intake and energy expenditure ${ }^{(1)}$. Higher energy intake is often related to poorer diet quality, which in turn is related to obesity ${ }^{(5,8)}$. In children, poor diet quality and behaviours might not only have immediate effects on health, but could also determine trajectories of eating habits that persist into adulthood ${ }^{(6,9)}$.

Dietary patterns and habits differ between ethnic groups living in the USA ${ }^{(10,11)}$ and among those with differing social backgrounds. This might lead to both higher- and lowerquality diets among children and adolescents from various racial/ethnic minority groups ${ }^{(12-16)}$. Several factors may influence racial/ethnic differences in diet. Better diet quality in children, for example, is more common in families that 
are better educated, have a higher household income ${ }^{(16,17)}$ and have a lower parental $\mathrm{BMI}^{(17,18)}$. Previous studies of diet among Hispanics and groups of African origin at various ages found greater levels of acculturation to be associated with more unhealthy dietary practices, such as greater intakes of salty snacks and energy-dense foods and lower intake of fruit and vegetables ${ }^{(19-22)}$. These findings suggest that, for example, a traditional Latino or Hispanic diet may be protective against poor diet quality ${ }^{(19,20)}$. Other socio-cultural factors, such as general beliefs about weight, can also influence diet. If parents perceive their children as underweight (whether they are or not), these children are more likely to be pressured to eat ${ }^{(23)}$; similarly, a child's diet may be overly restricted if parents perceive their child to be overweight ${ }^{(24)}$.

A limitation of the published literature related to childhood dietary intake is that most US studies have not focused primarily on racial/ethnic differences, or have only studied one racial/ethnic group without comparison between groups or examination of mechanisms related to racial/ethnic differences in diet. To our knowledge, no studies have investigated which factors can explain ethnic differences in diet in a multi-ethnic population of children of pre-school age. The purpose of the current study was to describe racial/ethnic differences in obesity-disposing aspects of diet among pre-school children and to examine the explanatory role of maternal BMI, maternal immigrant status and maternal perception of child's weight.

\section{Methods}

\section{Participants and study design}

Study subjects were participants in Project Viva, a prospective, pre-birth cohort study ${ }^{(25)}$. Between April 1999 and July 2002, research assistants recruited women at their initial prenatal visit at Harvard Vanguard Medical Associates, a large multi-specialty group practice located in eastern Massachusetts. We previously reported details of recruitment and subsequent retention ${ }^{(25)}$.

Of the 2128 women who delivered a singleton live infant, 2109 had information on child race/ethnicity. Of them, 1579 children were eligible for 3-year in-person follow-up by virtue of the mother having completed the prenatal assessments and providing consent for her child to be followed up. At age 3 years, information on diet was received for 1286 children $(81.4 \%$ of 1579$)$. For the current analysis, we excluded 149 children who had medical conditions or allergies requiring a specific diet and eighty-four children with missing covariates. The present study included 898 singleton children for whom information on diet and covariates were available from the following racial/ethnic groups: white non-Hispanic, black non-Hispanic and Hispanic. Children with other race/ethnicity than outlined above ( $n$ 155) were excluded because their numbers would be too small to analyse separately.
We compared the 898 participants in the current analysis with the excluded 927 participants with comparable race/ethnicity (white non-Hispanic, black non-Hispanic and Hispanic) who delivered a live infant. The included participants had a higher proportion of maternal white race $(80 \% v .67 \% ; P<0.001)$ and graduate education (33\% v. 23\%; $P<0 \cdot 001)$, a slightly lower mean maternal BMI $\left(24.7 \mathrm{~kg} / \mathrm{m}^{2}\right.$ v. $\left.25 \cdot 2 \mathrm{~kg} / \mathrm{m}^{2} ; P=0.05\right)$ and paternal BMI $\left(26.4 \mathrm{~kg} / \mathrm{m}^{2}\right.$ v. $\left.26 \cdot 8 \mathrm{~kg} / \mathrm{m}^{2} ; P=0.04\right)$ and fewer foreign-born mothers ( $14 \%$ v. 20\%; $P=0 \cdot 001)$.

After obtaining informed consent we collected demographic and health history information via interviews and self-administered questionnaires. We performed in-person study visits with the mother during pregnancy and with both mother and child immediately after delivery, at 6 months and at 3 years after delivery. Mothers completed mailed questionnaires at 1 year and 2 years postpartum. Covariate data, such as household income, education and immigrant status, was also collected during the study, through in-person visits and from mailed questionnaires, as outlined above. Human subjects committees of Harvard Pilgrim Health Care, Brigham and Women's Hospital, and Beth Israel Deaconess Medical Center approved the study protocols.

\section{Measurements}

\section{Race/ethnicity}

We assessed race/ethnicity during the 3-year interview using the question: 'Which of the following best describes your child's race or ethnicity?' Mothers had a choice of one or more of the following racial/ethnic groups: Hispanic or Latina, white or Caucasian, black or African American, Asian or Pacific Islander, American Indian or Alaskan Native, and other (please specify). For the participants who chose the 'other' race/ethnicity, the specified responses were compared with the US census definitions for the other five races and ethnicities and reclassified when appropriate. For the present study we included only those participants who indicated one race/ethnicity: white only, black only or Hispanic only. Participants who indicated more than one race/ethnicity or who indicated Asian, American Indian or other race/ethnicity were excluded.

\section{Dietary quality and energy intake}

We chose our diet quality information on the basis of current knowledge of which food groups, nutrients and dietary behaviours are associated with increased or decreased risk for diseases of adolescence and adulthood, including weight gain, Fe-deficiency anaemia, diabetes, osteoporosis, CVD and cancer ${ }^{(26-31)}$.

At the 3-year visit, mothers completed a semi-quantitative FFQ, validated for use in pre-school children, regarding their child's diet during the previous month to estimate food and nutrient intakes ${ }^{(32)}$. It included questions regarding usual frequency of intake of specific food and drink items 
during the past month. Response categories for foods, except for drinks and snack foods, included: 'never' (coded as 0 per fortnight), 'less than once a week' (1), 'once a week' (2), '2-4 times a week' (6), 'nearly daily or daily' (14) and '2 or more times per day' (coded as 28 times per fortnight). We defined snack food as chips, crackers, Jello, cookies or brownies, cake or cupcake, pie, chocolate candy, and other candy. Response categories for drinks and snack foods included: 'never' (coded as 0 per fortnight), 'less than once per week' (1), 'once per week' (2), '2-4 times per week' (6), 'nearly daily or daily' (14), '2-4 times per day' (42) and ' 5 or more times per day' (coded as 70 times per fortnight). Fast-food intake was defined by the question: 'In the past month, on average, how often did your child eat something from a fast food restaurant? (McDonald's, Burger King, Taco Bell, etc.)'. Response categories included: 'never/less than once per month' (coded as 0 times per fortnight), ' $1-3$ times per month' (1), 'once per week' (2), '2-4 times per week' (6), '5-6 times per week' (11) and 'once per day or more' (coded as 14 times per fortnight).

We estimated daily intakes of dietary fibre, dietary $\mathrm{Ca}$, fruit and vegetables, sugar-sweetened beverages, red and processed meats, percentages of total energy intake from total fat, trans-fat, saturated fat, polyunsaturated fat, carbohydrate and protein, and total energy intake $(\mathrm{kcal} / \mathrm{d})$. To calculate intakes of nutrients, we used the Harvard nutrient composition database developed for the Nurses' Health Study and other large cohort studies ${ }^{(33)}$.

\section{Potential explanatory factors}

We examined three potential explanatory factors: (i) maternal BMI; (ii) maternal immigrant status; and (iii) maternal perception of child's weight. At enrolment, mothers reported their pre-pregnancy weight and height as well as fathers' weight and height, from which we calculated BMI $\left(\mathrm{kg} / \mathrm{m}^{2}\right)$. We defined immigrant status of the mother by country of birth (US born or foreign born). To assess mother's perception of their child's weight status, we asked mothers to complete the following question from the 3-year questionnaire, 'How would you classify your child's current weight?', by choosing one of the following five responses: 'markedly underweight', 'underweight', 'average', 'overweight' or 'markedly overweight'.

\section{Other measures}

Sociodemographic characteristics included maternal prepregnancy age, education (less than Bachelor of Arts/ Bachelor of Science, Bachelor of Arts/Bachelor of Science or graduate degree), annual household income ( $<$ \$US 70000 or $\geq \$$ US 70000 ) and marital status (married/cohabitating or single). We obtained data on all sociodemographic characteristics at mothers' enrolment, except for household income, which we collected using the 3-year questionnaire. At 3 years, we measured children's height and weight using a calibrated stadiometer (Shorr Productions, Olney, MD,
USA) and scale (Seca model 881; Seca Corp., Hanover, MD, USA). We calculated age- and sex-specific BMI Z-scores using US national reference data ${ }^{(34)}$.

\section{Statistical analysis}

We first investigated unadjusted differences between ethnic groups with $\chi^{2}$ tests (categorical data), ANOVA (continuous data) or Kruskal-Wallis tests (food servings data). We then examined racial/ethnic differences in foods and food groups (count data) using negative binomial regression models rather than Poisson regression to account for overdispersion. We used linear regression to examine racial/ ethnic differences in nutrient intakes. We built our models in the following steps: first, we performed analyses of race/ ethnicity with dietary factors adjusted for child age, sex and total energy intake (model 1). Second, we adjusted for sociodemographic characteristics in model 2 including maternal age, education, annual household income and marital status. If we detected racial/ethnic differences in model 2 $(P \leq 0 \cdot 05)$, we additionally added maternal BMI (model 3 ), maternal immigrant status (model 4) or maternal perception of offspring's weight (underweight, average or overweight; model 5) to model 2 to help explain any association between ethnicity and foods or nutrients identified in model 2. We considered attenuation of estimated rate ratios (RR), for foods, or differences, for nutrients, of greater than $10 \%$ to indicate confounding (model 2) or mediation (model 3-5). In either case, such attenuation suggests that the potential confounder or mediator can (partially) explain racial/ethnic differences in food or nutrient intake.

In the regression models, the referent group was white children. We conducted all analyses using R $2 \cdot 14 \cdot 0^{(35)}$; negative binomial regressions used the MASS package ${ }^{(36)}$. We report adjusted rate ratios for foods and differences for intakes with $95 \%$ confidence intervals.

\section{Results}

\section{Characteristics of the sample}

Table 1 presents sample characteristics, according to child race/ethnicity. There were 723 white, 128 black and forty-seven Hispanic children. In unadjusted analyses, white mothers had a lower pre-pregnancy BMI, were older, had higher education and higher annual household income, and a larger proportion of non-single mothers $(P \leq 0 \cdot 001)$. No racial/ethnic differences were found in paternal BMI. A higher proportion of Hispanic mothers was born outside the USA (44.7\%) and perceived their child as underweight $(10 \cdot 6 \%)$ or overweight $(10 \cdot 6 \%)$ as compared with white (born outside USA $9 \cdot 2 \%$; perceived child underweight $7 \cdot 6 \%$; perceived child overweight $0 \cdot 8 \%$ ) and black mothers (born outside USA $30.4 \%$; perceived child underweight $5.4 \%$; perceived child overweight $0 \cdot 8 \% ; P \leq 0 \cdot 001$ ). The children of white non-Hispanic origin were generally younger $(P<0 \cdot 001)$. All children had a comparable BMI. 
Table 1 Selected parent and child characteristics according to child race/ethnicity: data from 898 mother-child pairs from the Project Viva prospective pre-birth cohort study, Boston, MA, USA

\begin{tabular}{|c|c|c|c|c|c|c|c|}
\hline & \multicolumn{2}{|c|}{$\begin{array}{l}\text { White, non-Hispanic } \\
\qquad(n 723)\end{array}$} & \multicolumn{2}{|c|}{$\begin{array}{l}\text { Black, non-Hispanic } \\
\qquad(n 128)\end{array}$} & \multicolumn{2}{|c|}{$\begin{array}{l}\text { Hispanic } \\
(n \text { 47) }\end{array}$} & \multirow[b]{2}{*}{$P$ value } \\
\hline & Mean or $n$ & SD or $\%$ & Mean or $n$ & SD or $\%$ & Mean or $n$ & SD or $\%$ & \\
\hline \multicolumn{8}{|l|}{ Sociodemographic characteristics } \\
\hline Maternal age (years) & $33 \cdot 3$ & $4 \cdot 3$ & $30 \cdot 2$ & $6 \cdot 4$ & $29 \cdot 4$ & $5 \cdot 2$ & $<0.001$ \\
\hline Maternal education, $n(\%)$ & & & & & & & $<0.001$ \\
\hline Less than BA or BS & 147 & $20 \cdot 3$ & 76 & $59 \cdot 4$ & 32 & $68 \cdot 1$ & \\
\hline $\mathrm{BA}$ or $\mathrm{BS}$ & 301 & $41 \cdot 7$ & 38 & $29 \cdot 7$ & 6 & $12 \cdot 8$ & \\
\hline Graduate degree & 275 & $38 \cdot 0$ & 14 & $10 \cdot 9$ & 9 & $19 \cdot 1$ & \\
\hline Annual household income $>\$$ US $70000, n(\%)$ & 545 & $75 \cdot 3$ & 38 & $29 \cdot 7$ & 14 & $29 \cdot 8$ & $<0.001$ \\
\hline Married/cohabiting, $n(\%)$ & 709 & $98 \cdot 0$ & 98 & $76 \cdot 5$ & 41 & $87 \cdot 2$ & $<0.001$ \\
\hline \multicolumn{8}{|l|}{ Maternal immigration status } \\
\hline Non-US born, $n(\%)$ & 67 & $9 \cdot 2$ & 39 & $30 \cdot 4$ & 21 & $44 \cdot 7$ & $<0.001$ \\
\hline \multicolumn{8}{|l|}{ Parental BMI } \\
\hline Maternal pre-pregnancy BMI $\left(\mathrm{kg} / \mathrm{m}^{2}\right)$ & $24 \cdot 1$ & $4 \cdot 7$ & $27 \cdot 4$ & $7 \cdot 0$ & $25 \cdot 7$ & $5 \cdot 7$ & $<0.001$ \\
\hline Paternal BMI $\left(\mathrm{kg} / \mathrm{m}^{2}\right)$ & $26 \cdot 3$ & $3 \cdot 6$ & $26 \cdot 6$ & $4 \cdot 7$ & $26 \cdot 9$ & $3 \cdot 4$ & 0.45 \\
\hline Mother's perception of offspring's weight, $n(\%)$ & & & & & & & $<0.001$ \\
\hline Underweight & 55 & $7 \cdot 6$ & 7 & $5 \cdot 4$ & 5 & $10 \cdot 6$ & \\
\hline Average & 662 & $91 \cdot 5$ & 120 & $93 \cdot 7$ & 37 & $78 \cdot 7$ & \\
\hline Overweight & 6 & $0 \cdot 8$ & 1 & $0 \cdot 8$ & 5 & $10 \cdot 6$ & \\
\hline \multicolumn{8}{|l|}{ Child characteristics } \\
\hline Age (months) & $38 \cdot 2$ & $2 \cdot 5$ & $39 \cdot 2$ & 3.9 & $39 \cdot 0$ & $3 \cdot 4$ & $<0.001$ \\
\hline Boy, $n(\%)$ & 362 & $50 \cdot 0$ & 71 & $55 \cdot 4$ & 31 & $66 \cdot 0$ & 0.07 \\
\hline BMI Z-score at age 3 years & 0.45 & 0.98 & 0.57 & $1 \cdot 04$ & 0.64 & $1 \cdot 24$ & 0.26 \\
\hline BMI percentile at age 3 years, $n(\%)$ & & & & & & & $0 \cdot 23$ \\
\hline $\mathrm{BMI}<85$ th percentile & 488 & $74 \cdot 1$ & 80 & $70 \cdot 2$ & 28 & $68 \cdot 3$ & \\
\hline BMI 85-95th percentile & 120 & $18 \cdot 2$ & 21 & $18 \cdot 4$ & 6 & $14 \cdot 6$ & \\
\hline BMI $\geq 95$ th percentile & 51 & $7 \cdot 7$ & 13 & $11 \cdot 4$ & 7 & $17 \cdot 1$ & \\
\hline
\end{tabular}

BA, Bachelor of Arts; BS, Bachelor of Science.

${ }^{*} \chi^{2}$ test for categories, ANOVA for age and BMI.

Table 2 Intakes of foods or food groups and nutrients according to child race/ethnicity: data from 898 mother-child pairs from the Project Viva prospective pre-birth cohort study, Boston, MA, USA

\begin{tabular}{|c|c|c|c|c|c|c|c|}
\hline \multirow[b]{2}{*}{ Food or food group } & \multicolumn{2}{|c|}{ White non-Hispanic } & \multicolumn{2}{|c|}{ Black non-Hispanic } & \multicolumn{2}{|c|}{ Hispanic } & \multirow[b]{2}{*}{$P$ value* } \\
\hline & Median & $\begin{array}{l}\text { 25th, } 75 \text { th } \\
\text { percentile }\end{array}$ & Median & $\begin{array}{l}\text { 25th, 75th } \\
\text { percentile }\end{array}$ & Median & $\begin{array}{l}\text { 25th, 75th } \\
\text { percentile }\end{array}$ & \\
\hline Sugar-sweetened beverages (svgs/d) & 0.07 & $0.00,0.29$ & $0 \cdot 50$ & $0.14,0.95$ & $0 \cdot 43$ & $0 \cdot 14,0 \cdot 64$ & $<0.001$ \\
\hline Fruit juice (svgs/d) & $1 \cdot 43$ & $0 \cdot 86,3 \cdot 00$ & $1 \cdot 43$ & $0 \cdot 86,3 \cdot 07$ & $2 \cdot 00$ & $0 \cdot 86,3 \cdot 29$ & 0.09 \\
\hline Skimmed or $1 \%$ dairy (svgs/d) & $1 \cdot 00$ & $0.43,3.43$ & 0.43 & $0 \cdot 14,1 \cdot 00$ & $0 \cdot 43$ & $0.43,0.71$ & $<0.001$ \\
\hline Whole or $2 \%$ dairy (svgs/d) & $2 \cdot 00$ & $1 \cdot 07,3 \cdot 86$ & $1 \cdot 96$ & $1 \cdot 14,3 \cdot 85$ & 3.57 & $1 \cdot 43,4.57$ & 0.03 \\
\hline Fast-food intake (svgs/month) & $2 \cdot 17$ & $0 \cdot 00,2 \cdot 17$ & $2 \cdot 17$ & $2 \cdot 17,4 \cdot 35$ & $2 \cdot 17$ & $2 \cdot 17,4 \cdot 35$ & $<0.001$ \\
\hline Snack-food intake (svgs/d) & $1 \cdot 50$ & $0 \cdot 93,2 \cdot 14$ & $1 \cdot 32$ & $0 \cdot 86,1 \cdot 80$ & $1 \cdot 50$ & $0 \cdot 86,2 \cdot 17$ & $0 \cdot 12$ \\
\hline Fruit and vegetable intake (svgs/d) & $4 \cdot 07$ & $2 \cdot 71,5 \cdot 36$ & $4 \cdot 25$ & $2 \cdot 79,6 \cdot 09$ & $3 \cdot 79$ & $2 \cdot 36,6 \cdot 11$ & 0.37 \\
\hline Red and processed meat (svgs/d) & $0 \cdot 79$ & $0 \cdot 43,1 \cdot 14$ & 0.93 & $0.57,1.43$ & $1 \cdot 07$ & $0.64,1.46$ & $<0.001$ \\
\hline Nutrient & Mean & SD & Mean & SD & Mean & SD & $P$ value* \\
\hline Total energy intake $(\mathrm{kJ} / \mathrm{d})$ & 6987 & 1916 & 6966 & 2548 & 7966 & 3134 & 0.01 \\
\hline Total energy intake $(\mathrm{kcal} / \mathrm{d})$ & 1670 & 458 & 1665 & 609 & 1904 & 749 & 0.01 \\
\hline$\%$ of energy intake from total fat & $30 \cdot 1$ & $5 \cdot 0$ & $29 \cdot 9$ & $5 \cdot 0$ & $29 \cdot 6$ & $4 \cdot 3$ & 0.67 \\
\hline$\%$ of energy intake from trans-fat & $1 \cdot 17$ & $0 \cdot 34$ & $1 \cdot 12$ & $0 \cdot 33$ & $1 \cdot 07$ & $0 \cdot 29$ & 0.07 \\
\hline$\%$ of energy intake from saturated fat & $12 \cdot 9$ & $2 \cdot 8$ & $12 \cdot 2$ & $2 \cdot 8$ & $12 \cdot 6$ & $2 \cdot 5$ & 0.05 \\
\hline$\%$ of energy intake from polyunsaturated fat & $6 \cdot 2$ & $1 \cdot 3$ & $6 \cdot 6$ & $1 \cdot 7$ & $5 \cdot 9$ & $1 \cdot 0$ & 0.002 \\
\hline$\%$ of energy intake from carbohydrates & $55 \cdot 9$ & $6 \cdot 0$ & $56 \cdot 4$ & $6 \cdot 5$ & $55 \cdot 9$ & $5 \cdot 6$ & 0.73 \\
\hline$\%$ of energy intake from protein & $16 \cdot 0$ & $2 \cdot 2$ & $15 \cdot 6$ & $2 \cdot 4$ & $16 \cdot 0$ & $2 \cdot 1$ & $0 \cdot 17$ \\
\hline Dietary fibre $(g / d)$ & $13 \cdot 6$ & $3 \cdot 4$ & $14 \cdot 2$ & $4 \cdot 0$ & $13 \cdot 7$ & $3 \cdot 8$ & 0.31 \\
\hline $\mathrm{Ca}(\mathrm{mg} / \mathrm{d})$ & 1028 & 253 & 859 & 262 & 893 & 215 & $<0.001$ \\
\hline
\end{tabular}

svgs, servings.

*Kruskal-Wallis test for foods, ANOVA for nutrients.

Table 2 demonstrates intakes of foods or food groups and nutrients according to child race/ethnicity. Black and Hispanic children had higher intakes of sugar-sweetenend beverages, fast food and red/processed meats, and a lower intake of skimmed $/ 1 \%$ dairy (all $P<0 \cdot 001$ ). Whole dairy intake was highest among Hispanic children $(P=0 \cdot 03)$. 
Table 3 Racial/ethnic differences in rates of intake of foods and food groups (white non-Hispanic as reference group): data from 898 mother-child pairs from the Project Viva prospective pre-birth cohort study, Boston, MA, USA

\begin{tabular}{|c|c|c|c|c|c|c|c|c|c|c|}
\hline \multirow[b]{2}{*}{ Food or food group } & \multicolumn{2}{|c|}{ Model 1} & \multicolumn{2}{|c|}{ Model 2} & \multicolumn{2}{|c|}{ Model 3} & \multicolumn{2}{|c|}{ Model 4} & \multicolumn{2}{|c|}{ Model 5} \\
\hline & RR & $95 \% \mathrm{Cl}$ & $\mathrm{RR}$ & $95 \% \mathrm{Cl}$ & $\mathrm{RR}$ & $95 \% \mathrm{Cl}$ & $\mathrm{RR}$ & $95 \% \mathrm{Cl}$ & $\mathrm{RR}$ & $95 \% \mathrm{Cl}$ \\
\hline \multicolumn{11}{|c|}{ Sugar-sweetened beverages } \\
\hline White non-Hispanic & \multicolumn{2}{|r|}{$1 \cdot 00$} & \multicolumn{2}{|r|}{$1 \cdot 00$} & \multicolumn{2}{|c|}{$1 \cdot 00$} & \multicolumn{2}{|r|}{$1 \cdot 00$} & \multicolumn{2}{|r|}{$1 \cdot 00$} \\
\hline Black non-Hispanic & $3 \cdot 35^{*}$ & $2 \cdot 61,4 \cdot 37$ & $2 \cdot 59$ & $1.95,3.48$ & $2 \cdot 63$ & $1.97,3.54$ & $2 \cdot 52$ & $1 \cdot 87,3 \cdot 44$ & $2 \cdot 65$ & $2 \cdot 00,3 \cdot 57$ \\
\hline Hispanic & $2 \cdot 04$ & $1 \cdot 39,3 \cdot 14$ & $1 \cdot 59$ & $1 \cdot 07,2 \cdot 47$ & $1 \cdot 61$ & $1 \cdot 08,2 \cdot 49$ & $1 \cdot 57$ & $1 \cdot 05,2 \cdot 4$ & $1 \cdot 57$ & $1 \cdot 05,2.43$ \\
\hline \multicolumn{11}{|l|}{ Fruit juice } \\
\hline White non-Hispanic & & $1 \cdot 00$ & & $1 \cdot 00$ & \multirow{3}{*}{\multicolumn{2}{|c|}{$\dagger$}} & & & & \\
\hline Black non-Hispanic & $1 \cdot 11$ & $0.96,1 \cdot 29$ & $1 \cdot 04$ & $0 \cdot 87,1 \cdot 23$ & & & & & & \\
\hline Hispanic & $1 \cdot 10$ & $0.87,1 \cdot 39$ & $1 \cdot 03$ & $0 \cdot 82,1 \cdot 32$ & & & & & & \\
\hline \multicolumn{11}{|l|}{ Skimmed or $1 \%$ dairy } \\
\hline White non-Hispanic & \multicolumn{2}{|r|}{$1 \cdot 00$} & \multicolumn{2}{|r|}{$1 \cdot 00$} & \multicolumn{2}{|c|}{$1 \cdot 00$} & \multicolumn{2}{|r|}{1.00} & \multicolumn{2}{|r|}{1.00} \\
\hline Black non-Hispanic & 0.35 & $0.28,0.43$ & 0.42 & $0.33,0.53$ & 0.40 & $0.32,0.51$ & 0.41 & $0.33,0.53$ & 0.42 & $0.33,0.53$ \\
\hline Hispanic & 0.36 & $0.26,0.50$ & 0.43 & $0.31,0.61$ & 0.42 & $0.30,0.60$ & 0.42 & $0.30,0.60$ & 0.42 & $0.30,0.60$ \\
\hline \multicolumn{11}{|l|}{ Whole or $2 \%$ dairy } \\
\hline White non-Hispanic & \multicolumn{2}{|r|}{$1 \cdot 00$} & \multicolumn{2}{|r|}{$1 \cdot 00$} & & & & & & \\
\hline Black non-Hispanic & 0.96 & $0 \cdot 85,1 \cdot 10$ & 0.97 & $0 \cdot 84,1 \cdot 13$ & & & & & & \\
\hline Hispanic & $1 \cdot 06$ & $0.87,1 \cdot 31$ & $1 \cdot 07$ & $0 \cdot 87,1 \cdot 33$ & & & & & & \\
\hline \multicolumn{11}{|l|}{ Fast-food intake } \\
\hline White non-Hispanic & \multicolumn{2}{|r|}{$1 \cdot 00$} & \multicolumn{2}{|r|}{$1 \cdot 00$} & \multicolumn{2}{|r|}{$1 \cdot 00$} & & $1 \cdot 00$ & & $1 \cdot 00$ \\
\hline Black non-Hispanic & $1 \cdot 42$ & $1 \cdot 19,1 \cdot 70$ & $1 \cdot 28$ & $1 \cdot 05,1 \cdot 55$ & $1 \cdot 25$ & $1 \cdot 02,1 \cdot 52$ & $1 \cdot 38$ & $1 \cdot 12,1 \cdot 67$ & $1 \cdot 30$ & $1 \cdot 06,1 \cdot 58$ \\
\hline Hispanic & $1 \cdot 35$ & $1 \cdot 01,1 \cdot 76$ & $1 \cdot 22$ & $0.91,1.62$ & $1 \cdot 21$ & $0.90,1.60$ & $1 \cdot 33$ & $0.98,1.78$ & $1 \cdot 24$ & $0.92,1.65$ \\
\hline Snack-food intake & & & & & & & & & & \\
\hline White non-Hispanic & & $1 \cdot 00$ & & $1 \cdot 00$ & & $1 \cdot 00$ & & $1 \cdot 00$ & & $1 \cdot 00$ \\
\hline Black non-Hispanic & 0.89 & $0.81,0.95$ & 0.89 & $0 \cdot 80,1 \cdot 00$ & 0.88 & $0.79,0.99$ & 0.91 & $0.82,1.02$ & 0.89 & $0.80,1 \cdot 00$ \\
\hline Hispanic & 0.85 & $0.73,0.99$ & 0.83 & $0.72,0.98$ & 0.83 & $0.71,0.97$ & 0.87 & $0.74,1.02$ & 0.82 & $0.70,0.96$ \\
\hline Fruit and vegetable int & & & & & & & & & & \\
\hline White non-Hispanic & & $1 \cdot 00$ & & $1 \cdot 00$ & & & & & & \\
\hline Black non-Hispanic & $1 \cdot 07$ & $0.97,1 \cdot 18$ & $1 \cdot 10$ & $0.99,1.22$ & & & & & & \\
\hline Hispanic & 0.93 & $0.80,1.09$ & 0.97 & $0 \cdot 82,1 \cdot 14$ & & & & & & \\
\hline Red and processed me & & & & & & & & & & \\
\hline White non-Hispanic & & $1 \cdot 00$ & & $1 \cdot 00$ & & & & & & \\
\hline Black non-Hispanic & $1 \cdot 23$ & $1 \cdot 08,1 \cdot 40$ & $1 \cdot 11$ & $0 \cdot 96,1 \cdot 27$ & & & & & & \\
\hline Hispanic & $1 \cdot 17$ & $0.96,1.44$ & $1 \cdot 07$ & $0 \cdot 87,1 \cdot 32$ & & & & & & \\
\hline
\end{tabular}

$\mathrm{RR}$, rate ratio.

Data were analysed using negative binomial regression.

Model 1: adjusted for age, gender and total energy intake only; Model 2: model 1 and additionally adjusted for maternal age, education, marital status and annual household income; Model 3: model 2 and additionally adjusted for maternal BMI; Model 4: model 2 and additionally adjusted for immigrant status; Model 5: model 2 and additionally adjusted for maternal perception of child's weight.

*Bold font indicates a significant difference compared with the reference group.

tNo racial/ethnic differences found in model 2.

Hispanics also had the highest total energy intake $(P=0 \cdot 01)$. The percentage of energy intake from trans-fat and saturated fat was highest among whites (borderline significant). The percentage of energy intake from polyunsaturated fat was highest among blacks $(P=0 \cdot 002)$. Ca intake was highest among white children $(P<0 \cdot 001)$.

\section{Racial/ethnic differences in foods or food groups}

Table 3 presents data on racial/ethnic differences in intake of foods and food groups analysed with negative binomial regression. In model 1 (adjusted for age, sex and energy intake), we observed higher intakes of sugar-sweetened beverages and fast food among black ( $\mathrm{RR}=3.35(95 \% \mathrm{CI}$ $2 \cdot 61,4 \cdot 37)$ and $\mathrm{RR}=1 \cdot 42(95 \% \mathrm{CI} 1 \cdot 19,1 \cdot 70)$, respectively) and Hispanic (RR $=2 \cdot 04(95 \% \mathrm{CI} 1 \cdot 39,3 \cdot 14)$ and $\mathrm{RR}=1 \cdot 35$ (95\% CI 1.01, 1.76), respectively) children compared with white children. Both black and Hispanic children drank $\sim 65 \%$ less skimmed $/ 1 \%$ milk (black: $\mathrm{RR}=0.35(95 \%$ CI $0 \cdot 28,0 \cdot 43)$; Hispanic: $\mathrm{RR}=0.36(95 \%$ CI $0 \cdot 26,0 \cdot 50)$ ) and $10-15 \%$ less snack food (black: $\mathrm{RR}=0.89(95 \% \mathrm{CI}$ $0.81,0.95)$; Hispanic: $\mathrm{RR}=0.85(95 \%$ CI $0.73,0.99))$ than white children. Black children ate more red/processed meat $(\mathrm{RR}=1.23(95 \% \mathrm{CI} 1.08,1 \cdot 40))$ than white children (Table 3).

Sociodemographic characteristics confounded the observed racial/ethnic differences (Table 3; model 2). Adjustment for sociodemographic characteristics attenuated our observed rates for sugar-sweetened beverages $(\sim 12 \%)$, fast food ( $\sim 10 \%)$, red/processed meat (10\%) and skimmed/1\% milk $(\sim 20 \%)$ in black and Hispanic children. However, most differences remained significant except for the higher rates of fast food intake in Hispanic children and red/processed meat in black children (Table 3).

\section{Explanatory role of maternal BMI, immigrant status and perception of offspring's weight on foods or food groups}

The rate ratios for foods or food groups changed minimally after adding maternal BMI, immigrant status or perception of offspring's weight into the model; except for fast food when adjusting for maternal immigrant status (Table 3). Being a mother born in a foreign country was 
associated with lower fast-food rates (RR $=0 \cdot 80(95 \% \mathrm{CI}$ $0 \cdot 64,0.98)$ ) compared with US-born mothers. Test for interaction revealed that there was no interaction between ethnicity and maternal immigrant status for fast-food intake (likelihood ratio test: $P=0 \cdot 57$ ).

\section{Racial/etbnic differences in nutrient intake}

Table 4 presents data on racial/ethnic differences in intake of nutrients analysed with linear regression. After adjustment for age and gender (model 1), daily energy intake was 962 (95\% CI 322, 1602) kJ (230 (95\% CI 77, 383) kcal) higher in Hispanic children than in whites. Blacks consumed a lower percentage of total energy intake from saturated fat $(-0.67(95 \% \mathrm{CI}-1 \cdot 22,-0 \cdot 11) \%)$ and had higher energy intake from polyunsaturated fat (0.42 (95\% CI 0.16, 0.69) \%). Both blacks and Hispanics consumed less Ca daily (blacks: -166 (95\% CI -215 , -117) mg/d; Hispanics: - 115 (95\% CI - 192, -39) mg/d) compared with whites.

Sociodemographic characteristics confounded some of the observed racial/ethnic differences (Table 4; model 2). Adjustment for sociodemographic characteristics attenuated our observed estimates for total energy intake (16\%), percentage of energy intake from polyunsaturated fat (26\%), Ca intake $(\sim 40 \%)$ and differences in percentage of energy from trans-fat $(\sim 55 \%)$, saturated fat (28\%) and dietary fibre $(73 \%)$ became greater in black and Hispanic children compared with the whites.

\section{Explanatory role of maternal BMI, immigrant status and perception of offspring's weight on nutrients}

Racial/ethnic differences in nutrients changed minimally after adding maternal BMI (model 3), immigrant status (model 4) or perception of offspring's weight into the model (model 5). After controlling for maternal immigrant status we found that racial/ethnic differences became greater $(11 \%)$ for energy intake, but attenuated for percentage of energy intake from trans-fat ( 30\%), saturated fat (11.5\%) and dietary fibre (28.3\%; model 4). In general, being a mother born in a foreign country was associated with lower energy intake $(-234(95 \% \mathrm{CI}-661,192) \mathrm{kJ} / \mathrm{d}$ $(-56(95 \% \mathrm{CI}-158,46) \mathrm{kcal} / \mathrm{d}))$, lower percentage of energy intake from trans-fat $(-0 \cdot 11$ (95\% CI $-0 \cdot 18$, $-0.04) \%)$ and saturated fat $(-0.36(95 \% \mathrm{CI}-0.93,0 \cdot 22)$ $\%)$, and a higher intake of dietary fibre (0.94 (95\% CI 0.22, $1 \cdot 65) \mathrm{g} / \mathrm{d}$ ) in their 3 -year-old offspring's diet. Test for interaction revealed that there was no interaction between ethnicity and maternal immigrant status for these outcome measures (likelihood ratio test: $P>0 \cdot 32$ ).

\section{Discussion}

In the present cohort we found higher intakes of sugarsweetened bevarages and fast food and a lower intake of skimmed/1\% milk among black and Hipanic children compared with white children. Snack intake, on the other hand, tended to be smaller among these groups. Some nutrient intakes (total energy intake, percentage of energy from trans-fat and saturated fat, dietary fibre intake) were also different between racial/ethnic groups. Most racial/ethnic differences in diet quality remained independent of sociodemographic-related factors. Maternal BMI and perception of child's weight did not explain the observed differences in diet quality. Controlling for maternal immigrant status revealed that being born in a foreign country was associated with higher-quality intake of some nutrients and fast-food intake tended to be lower.

Some of the racial/ethnic differences in diet that we observed have been described in previous studies. For example, the higher intake of sugar-sweetenend beverages and lower intake of skimmed/1\% milk among Hispanic and especially black children of pre-school age is consistent with previous studies ${ }^{(37,38)}$. In general, higher intake of milk and lower intake of sugar-sweetened beverages is associated with improved nutrient intake (e.g. vitamin $\mathrm{A}, \mathrm{Ca}, \mathrm{K}$ ) and a healthier $\operatorname{diet}^{(38,39)}$. It is possible that the lower $\mathrm{Ca}$ intake we observed among black and Hispanic children was due to the lower intake of skimmed/ $1 \%$ milk they reported. It is also possible that the higher sugar-sweetened beverage and fast-food intakes could have contributed to the higher energy intake in Hispanics. While black children also had higher intakes of sugarsweetened beverages and fast food, we did not observe higher energy intakes among blacks. Although we cannot fully explain why, this suggests that the higher intakes of sugar-sweetened beverages and fast food in black children, which should lead to overall higher energy intake, is compensated by lower intakes of other energy-dense foods (e.g. lower snack-food intake). The healthier fat intake (in particular, the lower percentage of energy derived from saturated fat) among black children has been reported in an earlier study on adults ${ }^{(40)}$ and in a cohort study of 9-10-year-old children from the $\mathrm{UK}^{(12)}$.

The influence of socio-economic status on diet is well reported $^{(11,16,17,20,22,41-43)}$. Education level and household income, frequently used measures of socio-economic status, are associated with higher intakes of foods considered healthful such as dairy, fruit and vegetables ${ }^{(16,42)}$ and better nutritional status ${ }^{(16,22,41)}$. Better-educated parents might be more health conscious and have greater nutritional knowledge compared with less-educated parents. Furthermore, being married or cohabiting enhances family resources (e.g. income, time), whereas single mothers receive less social support which may negatively affect resources $^{(44)}$. In our study, adjusting for these characteristics of socio-economic status partially explained our observed racial/ethnic differences at baseline, but the observed diffferences remained even after adjustment for sociodemographic characteristics suggesting that other factors might play a role. 
Table 4 Racial/ethnic differences in intake of nutrients (white non-Hispanic as reference group): data from 898 mother-child pairs from the Project Viva prospective pre-birth cohort study, Boston, MA, USA

\begin{tabular}{|c|c|c|c|c|c|c|c|c|c|c|}
\hline \multirow[b]{2}{*}{ Nutrient } & \multicolumn{2}{|c|}{ Model 1} & \multicolumn{2}{|c|}{ Model 2} & \multicolumn{2}{|c|}{ Model 3} & \multicolumn{2}{|c|}{ Model 4} & \multicolumn{2}{|c|}{ Model 5} \\
\hline & $\beta$ & $95 \% \mathrm{Cl}$ & $\beta$ & $95 \% \mathrm{Cl}$ & $\beta$ & $95 \% \mathrm{Cl}$ & $\beta$ & $95 \% \mathrm{Cl}$ & $\beta$ & $95 \% \mathrm{Cl}$ \\
\hline \multicolumn{11}{|c|}{ Total energy intake (kJ/d) } \\
\hline White non-Hispanic & \multicolumn{2}{|r|}{0.00} & \multicolumn{2}{|r|}{0.00} & \multicolumn{2}{|r|}{0.00} & \multicolumn{2}{|r|}{0.00} & \multicolumn{2}{|r|}{0.00} \\
\hline Black non-Hispanic & -29 & $-439,381$ & -105 & $-565,356$ & -29 & $-498,435$ & -46 & $-519,431$ & -105 & $-565,356$ \\
\hline Hispanic & $962+$ & 322,1602 & 833 & 163,1506 & 854 & 188,1527 & 925 & 234,1615 & 841 & 163,1519 \\
\hline \multicolumn{11}{|c|}{ Total energy intake $(\mathrm{kcal} / \mathrm{d})$} \\
\hline White non-Hispanic & \multicolumn{2}{|r|}{0.00} & \multicolumn{2}{|r|}{0.00} & \multicolumn{2}{|r|}{0.00} & \multicolumn{2}{|r|}{0.00} & \multicolumn{2}{|r|}{0.00} \\
\hline Black non-Hispanic & -7 & $-105,91$ & -25 & $-135,85$ & -7 & $-119,104$ & -11 & $-124,103$ & -25 & $-135,85$ \\
\hline Hispanic & 230 & 77, 383 & 199 & 39,360 & 204 & 45,365 & 221 & 56,386 & 201 & 39, 363 \\
\hline \multicolumn{11}{|c|}{$\%$ of energy intake from total fat } \\
\hline White non-Hispanic & \multicolumn{2}{|r|}{0.00} & \multicolumn{2}{|r|}{0.00} & \multirow{3}{*}{\multicolumn{2}{|c|}{$\ddagger$}} & & & & \\
\hline Black non-Hispanic & -0.32 & $-1 \cdot 29,0.65$ & -0.99 & $-2 \cdot 08,0 \cdot 10$ & & & & & & \\
\hline Hispanic & -0.57 & $-2.09,0.94$ & $-1 \cdot 29$ & $-2 \cdot 87,0 \cdot 30$ & & & & & & \\
\hline \multicolumn{11}{|c|}{$\%$ of energy intake from trans-fat } \\
\hline White non-Hispanic & & 0.00 & & 0.00 & & 0.00 & & 0.00 & & 0.00 \\
\hline Black non-Hispanic & -0.06 & $-0 \cdot 12,0.01$ & $-0 \cdot 10$ & $-0.18,-0.03$ & $-0 \cdot 10$ & $-0.18,-0.03$ & -0.07 & $-0 \cdot 15,0.00$ & $-0 \cdot 10$ & $-0.18,-0.03$ \\
\hline Hispanic & $-0 \cdot 10$ & $-0.20,0.00$ & -0.15 & $-0.26,-0.04$ & $-0 \cdot 15$ & $-0.26,-0.04$ & $-0 \cdot 11$ & $-0.22,0.00$ & -0.15 & $-0.26,-0.04$ \\
\hline$\%$ of energy intake fro & & & & & & & & & & \\
\hline White non-Hispanic & & 0.00 & & 0.00 & & 0.00 & & $0 \cdot 00$ & & 0.00 \\
\hline Black non-Hispanic & -0.67 & $-1 \cdot 22,-0.11$ & -0.86 & $-1.48,-0.23$ & -0.81 & $-1 \cdot 43,-0.18$ & -0.77 & $-1 \cdot 40,-0 \cdot 13$ & -0.86 & $-1.48,-0.24$ \\
\hline Hispanic & $-0 \cdot 32$ & $-1 \cdot 18,0.54$ & -0.51 & $-1.41,0.39$ & -0.49 & $-1 \cdot 40,0.41$ & -0.37 & $-1 \cdot 30,0.56$ & -0.43 & $-1 \cdot 33,0 \cdot 49$ \\
\hline$\%$ of energy intake fro & & & & & & & & & & \\
\hline White non-Hispanic & & 0.00 & & 0.00 & & & & & & \\
\hline Black non-Hispanic & 0.42 & $0.16,0.69$ & 0.27 & $-0.03,0.57$ & & & & & & \\
\hline Hispanic & $-0 \cdot 21$ & $-0.63,0.20$ & -0.38 & $-0.81,0.05$ & & & & & & \\
\hline$\%$ of energy intake fro & & & & & & & & & & \\
\hline White non-Hispanic & & 0.00 & & 0.00 & & & & & & \\
\hline Black non-Hispanic & 0.49 & $-0.71,1.69$ & 1.03 & $-0.31,2 \cdot 37$ & & & & & & \\
\hline Hispanic & $0 \cdot 12$ & $-1 \cdot 74,1.98$ & 0.66 & $-1 \cdot 28,2 \cdot 61$ & & & & & & \\
\hline$\%$ of energy intake fro & & & & & & & & & & \\
\hline White non-Hispanic & & 0.00 & & 0.00 & & & & & & \\
\hline Black non-Hispanic & -0.40 & $-0.84,0.03$ & $-0 \cdot 21$ & $-0.69,0.28$ & & & & & & \\
\hline Hispanic & 0.07 & $-0.60,0.74$ & $0 \cdot 31$ & $-0 \cdot 40,1 \cdot 01$ & & & & & & \\
\hline Dietary fibre $(g / d)^{\star}$ & & & & & & & & & & \\
\hline White non-Hispanic & & 0.00 & & 0.00 & & 0.00 & & 0.00 & & 0.00 \\
\hline Black non-Hispanic & 0.49 & $-0 \cdot 20,1 \cdot 19$ & 0.85 & $0.08,1.62$ & 0.85 & $0.07,1.64$ & $0 \cdot 61$ & $-0 \cdot 18,1 \cdot 41$ & 0.85 & $0.07,1.62$ \\
\hline Hispanic & -0.04 & $-0 \cdot 13,1 \cdot 04$ & 0.28 & $-0.84,1 \cdot 41$ & 0.29 & $-0.84,1 \cdot 41$ & -0.08 & $-1 \cdot 24,1 \cdot 07$ & $0 \cdot 31$ & $-0.83,1.45$ \\
\hline $\mathrm{Ca}(\mathrm{mg} / \mathrm{d})^{\star}$ & & & & & & & & & & \\
\hline White non-Hispanic & & $0 \cdot 00$ & & 0.00 & & 0.00 & & 0.00 & & 0.00 \\
\hline Black non-Hispanic & -166 & $-215,-117$ & -120 & $-175,-65$ & -118 & $-173,-63$ & -129 & $-185,-73$ & -120 & $-175,-66$ \\
\hline Hispanic & -115 & $-192,-39$ & -67 & $-147,11$ & -67 & $-146,-13$ & -81 & $-163,1$ & -67 & $-148,13$ \\
\hline
\end{tabular}


Our study and the existing literature have shown that less acculturation is associated with more healthful dietary intake and behaviours in the USA ${ }^{(19-21,45,46)}$. For example, the traditional Hispanic or Latino diet is rich in low-fat foods such as beans, tortillas and rice ${ }^{(47)}$. African diets generally contain more rice, fruit, vegetables and fish, compared with the Western diet ${ }^{(21,45)}$. In our study, being a mother born outside the USA was associated with some higher-quality nutrient intakes and lower rates of fast food. According to the theory of Korturk-Runefors, changes in food habits begin with accessory foods (e.g. drinks and snacks) that are often not considered as 'real' food ${ }^{(48,49)}$. Dinner meals, which are strongly associated with cultural identity, may be the last to change. Our results showed that higher intake of sugar-sweetened drinks was independent of immigrant status. Since all foreign-born mothers in our study had lived in the USA for at least 4 years (all children were born in the USA), probably some dietary transition has already taken place.

Maternal BMI did not explain the observed racial/ethnic differences in diet quality. However, there was a strong association between sociodemographic characteristics, such as education level, and maternal BMI. Since these covariates were already included in the model before maternal BMI was added, they may have explained some of the difference expected to be associated with maternal BMI. We also hypothesized that perception of weight could explain racial/ethnic differences in diet. Although the association between maternal perception of offspring's weight and diet in children is not often studied, MacFarlane et al. described in 12-15-year-olds that parental concern about adolescents' weight was associated with lower intakes of energy-dense snacks ${ }^{(24)}$. Furthermore, general beliefs about children's weight differs among ethnic groups in the USA ${ }^{(50-52)}$. Possibly these effects on dietary intake are not yet present at pre-school age since the previous study was done in adolescents.

Strengths of the present study included detailed dietary information using validated tools and the ability to compare racial/ethnic groups for differences in diet quality instead of comparing with previous studies or population estimates. We were able to adjust for several important confounders and formulate the role of important determinants. The study also had several potential limitations. First, maternal BMI was based on self-reported height and weight, and the mothers also reported paternal weight and height. Self-reported height tends to be slightly overestimated and weight underestimated, resulting in an underestimation of $\mathrm{BMI}^{(53)}$. Furthermore, white adults are more likely than blacks and Hispanics to under-report BMI, which suggests that the racial/ethnic differences in maternal BMI might be slightly overestimated in our study ${ }^{(54)}$. Second, the sample was not fully representative for the general population. For example, the education and income levels of our study population were relatively high. Additionally the sample was collected in an urban setting. Our results may not be generalizable to more socio-economically disadvantaged populations or outside urban settings. Third, the Harvard FFQ was validated only in Native American and Caucasian children. The intake of foods might be underestimated for black and Hispanic children due to lack of ethnic-specific food items in the questionnaire, although parents had the ability to include other foods their child eats at least once weekly in a separate section of the questionnaire. Fourth, nutrient intake and especially total energy intake are difficult to measure with FFQ. However, there is no evidence to assume that estimated errors would be different for the racial/ethnic groups, so our estimates of relative differences are likely to be accurate. Finally, due to small samples of blacks and Hispanics, the lack of significant differences in dietary intake might be due to a lack of statistical power. Alternatively, we might have underestimated the ethnic differences in diet due to selection.

\section{Conclusion}

In sum, our results suggest that there are several racial/ ethnic differences in the dietary intake of 3-year-olds. Compared with white children, black and Hispanic children ate more unhealthy foods such as sugar-sweetened beverages and less low-fat dairy. Total energy intake was substantially higher in Hispanic children; however, intakes of snack food (Hispanic children) and fat (black children) tended to be lower. Awareness of these racial/ ethnic differences in diet is of public health relevance since the US black and Hispanic population is not only large but represents groups that have higher prevalences of obesity and chronic diseases. Being a mother born outside the USA was associated with more healthful intakes of some nutrients and lower intake of fast food for her offspring. Because diet may affect weight gain or chronic diseases in later life, health professionals should encourage immigrant mothers to retain their traditional diets that contain less trans-fat and saturated fat and more dietary fibre.

\section{Acknowledgements}

Sources of funding: This study was supported by a grant from the National Institute on Minority Health and Health Disparities (MD 003963). Conflicts of interest: There is no conflict of interest. Authors' contributions: M.L.A.d.H. and E.M.T. developed the concept of the present study as part of Project Viva. M.L.A.d.H. conducted the analyses and K.P.K. provided statistical advice. All co-authors contributed to interpreting the results and writing the manuscript, and they have read and approved the final version. Acknowledgements: The authors especially thank all participants and staff of Project Viva for their time and involvement. 


\section{References}

1. Gortmaker SL, Swinburn BA, Levy D et al. (2011) Changing the future of obesity: science, policy, and action. Lancet 378, 838-847.

2. Ogden CL, Carroll MD, Kit BK et al. (2012) Prevalence of obesity and trends in body mass index among US children and adolescents, 1999-2010. JAMA 307, 483-490.

3. Singh GK, Kogan MD, Van Dyck PC et al. (2008) Racial/ ethnic, socioeconomic, and behavioral determinants of childhood and adolescent obesity in the United States: analyzing independent and joint associations. Ann Epidemiol 18, 682-695.

4. Skelton JA, Cook SR, Auinger P et al. (2009) Prevalence and trends of severe obesity among US children and adolescents. Acad Pediatr 9, 322-329.

5. Swinburn BA, Sacks G, Hall KD et al. (2011) The global obesity pandemic: shaped by global drivers and local environments. Lancet 378, 804-814.

6. Craigie AM, Lake AA, Kelly SA et al. (2011) Tracking of obesity-related behaviours from childhood to adulthood: a systematic review. Maturitas 70, 266-284.

7. Walker SE, Gurka MJ, Oliver MN et al. (2010) Racial/ethnic discrepancies in the metabolic syndrome begin in childhood and persist after adjustment for environmental factors. Nutr Metab Cardiovasc Dis 22, 141-148.

8. Moreno LA \& Rodriguez G (2007) Dietary risk factors for development of childhood obesity. Curr Opin Clin Nutr Metab Care 10, 336-341.

9. Dorgan JF, Liu L, Barton BA et al. (2011) Adolescent diet and metabolic syndrome in young women: results of the Dietary Intervention Study in Children (DISC) follow-up study. J Clin Endocrinol Metab 96, E1999-E2008.

10. Mennella JA, Ziegler P, Briefel R et al. (2006) Feeding infants and toddlers study: the types of foods fed to Hispanic infants and toddlers. I Am Diet Assoc 106, S96-S106.

11. Park SY, Murphy SP, Wilkens LR et al. (2005) Dietary patterns using the Food Guide Pyramid groups are associated with sociodemographic and lifestyle factors: the multiethnic cohort study. J Nutr 135, 843-849.

12. Donin AS, Nightingale CM, Owen CG et al. (2010) Nutritional composition of the diets of South Asian, black African-Caribbean and white European children in the United Kingdom: the Child Heart and Health Study in England (CHASE). Br J Nutr 104, 276-285.

13. Kleiser C, Mensink GB, Neuhauser H et al. (2010) Food intake of young people with a migration background living in Germany. Public Health Nutr 13, 324-330.

14. Lytle LA, Himes JH, Feldman H et al. (2002) Nutrient intake over time in a multi-ethnic sample of youth. Public Health Nutr 5, 319-328.

15. Troiano RP, Briefel RR, Carroll MD et al. (2000) Energy and fat intakes of children and adolescents in the United States: data from the national health and nutrition examination surveys. Am J Clin Nutr 72, 5 Suppl., 1343S-1353S.

16. Xie B, Gilliland FD, Li YF et al. (2003) Effects of ethnicity, family income, and education on dietary intake among adolescents. Prev Med 36, 30-40.

17. Fisk CM, Crozier SR, Inskip HM et al. (2011) Influences on the quality of young children's diets: the importance of maternal food choices. Br J Nutr 105, 287-296.

18. Ohlund I, Hernell O, Hornell A et al. (2010) BMI at 4 years of age is associated with previous and current protein intake and with paternal BMI. Eur J Clin Nutr 64, 138-145.

19. Batis C, Hernandez-Barrera L, Barquera S et al. (2011) Food acculturation drives dietary differences among Mexicans, Mexican Americans, and Non-Hispanic Whites. J Nutr 141, 1898-1906.
20. Dave JM, Evans AE, Saunders RP et al. (2009) Associations among food insecurity, acculturation, demographic factors, and fruit and vegetable intake at home in Hispanic children. J Am Diet Assoc 109, 697-701.

21. Desilets MC, Rivard M, Shatenstein B et al. (2007) Dietary transition stages based on eating patterns and diet quality among Haitians of Montreal, Canada. Public Health Nutr 10, 454-463.

22. Mazur RE, Marquis GS \& Jensen HH (2003) Diet and food insufficiency among Hispanic youths: acculturation and socioeconomic factors in the third National Health and Nutrition Examination Survey. Am J Clin Nutr 78, 1120-1127.

23. Francis LA, Hofer SM \& Birch LL (2001) Predictors of maternal child-feeding style: maternal and child characteristics. Appetite 37, 231-243.

24. Macfarlane A, Crawford D \& Worsley A (2010) Associations between parental concern for adolescent weight and the home food environment and dietary intake. J Nutr Educ Behav 42, 152-160.

25. Gillman MW, Rich-Edwards JW, Rifas-Shiman SL et al. (2004) Maternal age and other predictors of newborn blood pressure. J Pediatr 144, 240-245.

26. Gillman MW (1996) Enjoy your fruits and vegetables. BMJ 313, 765-766

27. Johnston CC Jr, Miller JZ, Slemenda CW et al. (1992) Calcium supplementation and increases in bone mineral density in children. $N$ Engl J Med 327, 82-87.

28. Oh K, Hu FB, Manson JE et al. (2005) Dietary fat intake and risk of coronary heart disease in women: 20 years of follow-up of the Nurses' Health Study. Am J Epidemiol 161, 672-679.

29. Posner BM, Cobb JL, Belanger AJ et al. (1991) Dietary lipid predictors of coronary heart disease in men. The Framingham Study. Arch Intern Med 151, 1181-1187.

30. Salmeron J, Manson JE, Stampfer MJ et al. (1997) Dietary fiber, glycemic load, and risk of non-insulin-dependent diabetes mellitus in women. JAMA 277, 472-477.

31. Willett WC (1994) Diet and health: what should we eat? Science 264, 532-537.

32. Blum RE, Wei EK, Rockett HR et al. (1999) Validation of a food frequency questionnaire in Native American and Caucasian children 1 to 5 years of age. Matern Child Health $J 3,167-172$.

33. Hu FB, Stampfer MJ, Manson JE et al. (1997) Dietary fat intake and the risk of coronary heart disease in women. N Engl J Med 337, 1491-1499.

34. National Center for Health Statistics (2010) Homepage. http://www.cdc.gov/growthcharts/ (accessed September 2010).

35. Venables WN \& Ripley BD (2002) Modern Applied Statistics with $S$, 4th ed. New York: Springer.

36. R Development Core Team (2011) $R$ : A Language and Environment for Statistical Computing. Vienna: R Foundation for Statistical Computing; available at http://www. r-project.org/

37. Dennison BA, Rockwell HL \& Nichols MJ (2001) Use of low-fat milk by children in the New York State WIC varies with parental characteristics. J Am Diet Assoc 101, 464-466.

38. O'Neil CE, Nicklas TA, Liu Y et al. (2009) Impact of dairy and sweetened beverage consumption on diet and weight of a multiethnic population of head start mothers. J Am Diet Assoc 109, 874-882.

39. LaRowe TL, Moeller SM \& Adams AK (2007) Beverage patterns, diet quality, and body mass index of US preschool and school-aged children. J Am Diet Assoc 107, 1124-1133.

40. Vyas A (2003) Nutrient intakes of an adult Pakistani, European and African-Caribbean community in inner city Britain. J Hum Nutr Diet 16, 327-337.

41. Crawford PB, Obarzanek E, Schreiber GB et al. (1995) The effects of race, household income, and parental education 
on nutrient intakes of 9- and 10-year-old girls. NHLBI Growth and Health Study. Ann Epidemiol 5, 360-368.

42. North K \& Emmett P (2000) Multivariate analysis of diet among three-year-old children and associations with socio-demographic characteristics. The Avon Longitudinal Study of Pregnancy and Childhood (ALSPAC) Study Team. Eur J Clin Nutr 54, 73-80.

43. Schefske SD, Bellows AC, Byrd-Bredbenner C et al. (2010) Nutrient analysis of varying socioeconomic status home food environments in New Jersey. Appetite 54, 384-389.

44. Elfhag K \& Rasmussen F (2008) Food consumption, eating behaviour and self-esteem among single $v$. married and cohabiting mothers and their 12-year-old children. Public Health Nutr 11, 934-939.

45. Delisle H (2010) Findings on dietary patterns in different groups of African origin undergoing nutrition transition. Appl Physiol Nutr Metab 35, 224-228.

46. Satia-Abouta J, Patterson RE, Neuhouser ML et al. (2002) Dietary acculturation: applications to nutrition research and dietetics. J Am Diet Assoc 102, 1105-1118.

47. Gordon-Larsen P, Harris KM, Ward DS et al. (2003) Acculturation and overweight-related behaviors among
Hispanic immigrants to the US: the National Longitudinal Study of Adolescent Health. Soc Sci Med 57, 2023-2234.

48. Kocturk T (1995) Structure and change in food habits. Scand J Nutr 39, 2-4.

49. Mellin-Olsen T \& Wandel M (2005) Changes in food habits among Pakistani immigrant women in Oslo, Norway. Ethn Health 10, 311-339.

50. Dammann KW, Smith C \& Richards R (2011) Low-income minority and homeless mothers' perceptions of their 9-13 year-old children's weight status, diet, and health. Matern Child Health J 15, 106-114.

51. Dixon B, Pena MM \& Taveras EM (2012) Lifecourse approach to racial/ethnic disparities in childhood obesity. Adv Nutr 3, 73-82.

52. Killion L, Hughes SO, Wendt JC et al. (2006) Minority mothers' perceptions of children's body size. Int J Pediatr Obes 1, 96-102.

53. Stewart AW, Jackson RT, Ford MA et al. (1987) Underestimation of relative weight by use of self-reported height and weight. Am J Epidemiol 125, 122-126.

54. Wen M \& Kowaleski-Jones L (2012) Sex and ethnic differences in validity of self-reported adult height, weight and body mass index. Ethn Dis 22, 72-78. 